\title{
COCHRANE
} CORNER

${ }^{\dagger}$ This review is an abridged version of a Cochrane Review previously published in the Cochrane Database of Systematic Reviews, 2014, Issue 4, doi: 10.1002/14651858.CD010646. pub2 (see www.thecochranelibrary. com for information). Cochrane Reviews are regularly updated as new evidence emerges and in response to feedback, and the Cochrane Database of Systematic Reviews should be consulted for the most recent version of the review.

We thank the Cochrane Review Group for their support in publishing these reviews.

\section{Cognitive behavioural therapy (brief versus standard duration) for schizophrenia}

Farooq Naeem, Saeed Farooq \& David Kingdon

\section{Background}

Cognitive-behavioural therapy (CBT) is a psychotherapeutic approach that establishes links between thoughts, emotions and behaviours, and identifies and challenges dysfunctional thoughts. There is some evidence to suggest that CBT for people with psychosis (CBT-P) might also be an effective treatment for people with schizophrenia. There are, however, limitations in its provision owing to available resource and training issues. One way to tackle this problem might be to offer a brief version of CBT-P.

\section{Objectives}

To review the effects of brief CBT-P (6 to 10 regular sessions given in less than 4 months and using a manual) compared with standard CBT-P (12 to 20 regular sessions given in 4 to 6 months and using a manual) for people with schizophrenia.

\section{Search methods}

We searched the Cochrane Schizophrenia Group's Trials Register (August 21, 2013), which is based on regular searches of CINAHL, BIOSIS, AMED, Embase, PubMed, Medline, PsycINFO and registries of clinical trials. There are no language, date, document type, or publication status limitations for inclusion of records in the register. We inspected all references of the selected articles for further relevant trials. We also contacted experts in the field regarding brief CBT-P studies.

\section{Selection criteria}

Randomised controlled trials involving adults with schizophrenia or related disorders that compared brief CBT-P with standard CBT-P.

\section{Data collection and analysis}

Two review authors independently screened and assessed studies for inclusion using predetermined inclusion criteria.

\section{Main results}

We found only seven studies which used a brief version of CBT-P, but no study compared brief CBT-P with CBT-P of standard duration. Therefore, no studies could be included.

\section{Authors' conclusions}

There is currently no literature available that compares brief with standard CBT-P for people with schizophrenia. We cannot, therefore, conclude whether brief CBT-P is as effective, less effective or even more effective than standard courses of the same therapy. This lack of evidence for brief CBT-P has serious implications for research and practice. Well-planned, conducted and reported randomised trials are indicated.

Assessed as up to date: 7 December, 2012

See more at: http://summaries.cochrane.org/CD010646/brief-versusstandard-cognitive-behavioural-therapy-for-schizophrenia 